\title{
Experimental Demonstration of a Cognitive Optical Network for Reduction of Restoration Time
}

Kachris, Christoforos; Klonidis, Dimitris; Francescon, Antonio; Siracusa, Domenico; Salvadori, Elio; Barroso, Ramón J. Durán; de Miguel, Ignacio; Borkowski, Robert; Caballero Jambrina, Antonio; Tafur Monroy, Idelfonso

Total number of authors:

13

Published in:

2014 Optical Fiber Communications Conference and Exhibition (OFC)

Link to article, DOI:

10.1364/OFC.2014.W2A.28

Publication date:

2014

Document Version

Publisher's PDF, also known as Version of record

Link back to DTU Orbit

Citation (APA):

Kachris, C., Klonidis, D., Francescon, A., Siracusa, D., Salvadori, E., Barroso, R. J. D., de Miguel, I., Borkowski, R., Caballero Jambrina, A., Tafur Monroy, I., Ye, Y., Tymecki, A., \& Tomkos, I. (2014). Experimental Demonstration of a Cognitive Optical Network for Reduction of Restoration Time. In 2014 Optical Fiber Communications Conference and Exhibition (OFC) [W2A.28] IEEE. https://doi.org/10.1364/OFC.2014.W2A.28

\section{General rights}

Copyright and moral rights for the publications made accessible in the public portal are retained by the authors and/or other copyright owners and it is a condition of accessing publications that users recognise and abide by the legal requirements associated with these rights.

- Users may download and print one copy of any publication from the public portal for the purpose of private study or research.

- You may not further distribute the material or use it for any profit-making activity or commercial gain

- You may freely distribute the URL identifying the publication in the public portal 


\title{
Experimental Demonstration of a Cognitive Optical Network for Reduction of Restoration Time
}

\author{
Christoforos Kachris ${ }^{1}$, Dimitris Klonidis ${ }^{1}$, Antonio Francescon ${ }^{2}$, Domenico Siracusa ${ }^{2}$, Elio Salvadori ${ }^{2}$, Natalia \\ Fernández ${ }^{3}$, Tamara Jiménez ${ }^{3}$, Ramón J. Durán ${ }^{3}$, Ignacio de Miguel ${ }^{3}$, Juan Carlos Aguado ${ }^{3}$, Rubén M. \\ Lorenzo $^{3}$, Robert Borkowski ${ }^{4}$, Antonio Caballero ${ }^{4}$, Idelfonso Tafur Monroy ${ }^{4}$, Yabin Ye $^{6}$, Andrzej Tymecki ${ }^{5}$, \\ Ioannis Tomkos ${ }^{1}$ \\ ${ }^{1}$ Athens Information Technology, Greece \\ ${ }^{2}$ CREATE-NET, Italy \\ ${ }^{3}$ Universidad de Valladolid, Spain \\ ${ }^{4}$ Technical University of Denmark, Denmark \\ ${ }^{5}$ Orange Polska, Poland, ${ }^{6}$ Huawei Technologies, Germany
}

\begin{abstract}
This paper presents the implementation and performance evaluation of a cognitive heterogeneous optical network testbed. The testbed integrates the CMP, the data plane and the cognitive system and reduces by $48 \%$ the link restoration time.

OCIS codes: (060.4261) Networks, protection and restoration; (060.4250) Networks; (060.4510) Optical communications
\end{abstract}

\section{Introduction to Cognitive Reconfigurable Optical Networks}

Optical networks are facing increased levels of heterogeneity, from types of services to transmission technologies. Hence, a key issue of highly heterogeneous networks is how to efficiently control and manage network resources while fulfilling user demands and complying with quality of service requirements. A solution for such a scenario may come from cognitive networks. A cognitive network is defined as "A network with a process that can perceive current network conditions, and then plan, decide, and act on those conditions. The network can learn from these adaptations and use them to make future decisions, all while taking into account end-to-end goals.” [1]. Hence, there are three main ingredients in such a network:

- Monitoring elements, which provide the network with the perception of the current conditions, and thus enable an aware network.

- Software adaptable elements, which provide the network with the capacity of modifying its current configuration, thus enabling an adaptive network.

- Cognitive processes, which learn or make use of past history, so that even when facing two equivalent scenarios, the network (or the element containing those cognitive processes) may act in a different way if its previous history is different. This third element is the main feature which enables a cognitive network.

Therefore, a cognitive network is a network which is able to adapt itself to current or forecasted conditions by taking into account previous history, and which is able to act proactively, rather than reactively, in order to avoid problems before they arise. Moreover, those tasks should be performed autonomously, with little or no intervention of the network operator. Cognitive networks have already shown their advantages in wireless environments [2], but they are also applicable to wired communication architectures, and are especially appealing for optimizing performance in heterogeneous networks. The design of a cognitive optical network involves determining how the three aforementioned ingredients are implemented (and where), how they are glued together, as well as determining which tasks are going to be solved with the help of cognition.

The CHRON project [3] has proposed a novel architecture for cognitive heterogeneous reconfigurable optical networks. The aim of the CHRON project is to develop and showcase network architecture and a control plane which efficiently use resources in a heterogeneous scenario while fulfilling QoS requirements of each type of services and applications supported by the network. This cognitive network observes acts, learns and optimizes its performance, taking into account its high degree of heterogeneity with respect to QoS, transmission and switching techniques. For that aim, CHRON relies on cognition, so that control decisions must be made with an appropriate knowledge of current status, and supported by a learning process to improve performance with acquired experience. The physical layer of such a network reflects the current and upcoming situation faced by network operators, with high level of heterogeneity of physical interfaces and transmission systems such as modulation formats, wavelength capacity or coherent and non-coherent techniques.

The core element of the CHRON cognitive architecture is the cognitive decision system (CDS). The CDS receives traffic demands, and determines how to handle them by taking into account both the current status of the network and past history, and instructs the control plane to configure network elements accordingly. Therefore, the 
CDS is complemented with a network monitoring system, which provides traffic status and optical quality of transmission measurements, and with a set of GMPLS-based control and management mechanisms to implement the decisions that are made by the CDS and to disseminate the monitored information. The CDS is involved in very diverse tasks related to network control and optimization. Hence, rather than implementing the whole CDS as a monolithic module, it is divided into different modules, each offering a functionality (or a set of related functionalities), and all of them exploiting cognition. The advantages of the CDS have been shown in the past in several network topologies [4][4]. However, the CDS has been only validated in simulation mode.

\section{Cognitive Heterogeneous Reconfigurable Optical Network Testbed}

To evaluate the concepts of the CHRON project, a physical testbed has been implemented that demonstrates the advantages of a cognitive engine in optical networks. The physical testbed consists of three basic elements: the Cognitive Decision System (CDS), the Control and Management Plane (CMP) and the Data Plane, as it is depicted in Figure 1. The control plane is distributed and it is hosted in 4 nodes (4 separate PCs that are emulated 4 real network nodes) [5]. One separate machine is used to host the CDS, which performs all the required actions (routing decisions, storing of database, etc.).

The data plane hosts 4 nodes for the performance evaluation of the CHRON system. Each of the nodes consists of an Optical Cross Connect (OXC) module and the OXC controller that is used to communicate with the control plane. The Optical Cross Connects modules are actually 8x8 optical MEMS switches [7]. These optical MEMS consist of 64 mirrors that provide strictly non-blocking connection of any of 8 inputs to any of 8 outputs, reconfiguring up to 8 fiber ports. The optical MEMS switch functions independently of the wavelength, data rate or format and hence can support heterogeneous optical networks. The switching time of these MEMS switches is less than 10 ms. The MEMS switches are controlled using a special serial interface that is used to control each of the 64 mirrors. To control these optical switches, a low-cost Xilinx FPGA board is used that accepts the commands from the control nodes and configures the optical switches.

To evaluate the CHRON system, an FPGA-based traffic generator has been used. The FPGA board is based on the Altera Transceiver Signal Integrity Development board [8]. This board hosts six full-duplex transceiver channels routed to SMA connectors that can support up to $11.3 \mathrm{Gbps}$ data rates. The transceivers generate and check several pseudo-random binary sequence (PRBS) patterns and are reconfigured dynamically to change the differential output voltage, the pre-emphasis, and the equalization settings based on the network requirements. These transceivers are used to modulate the 6 OOK channels that are used in the current implementation of the testbed.

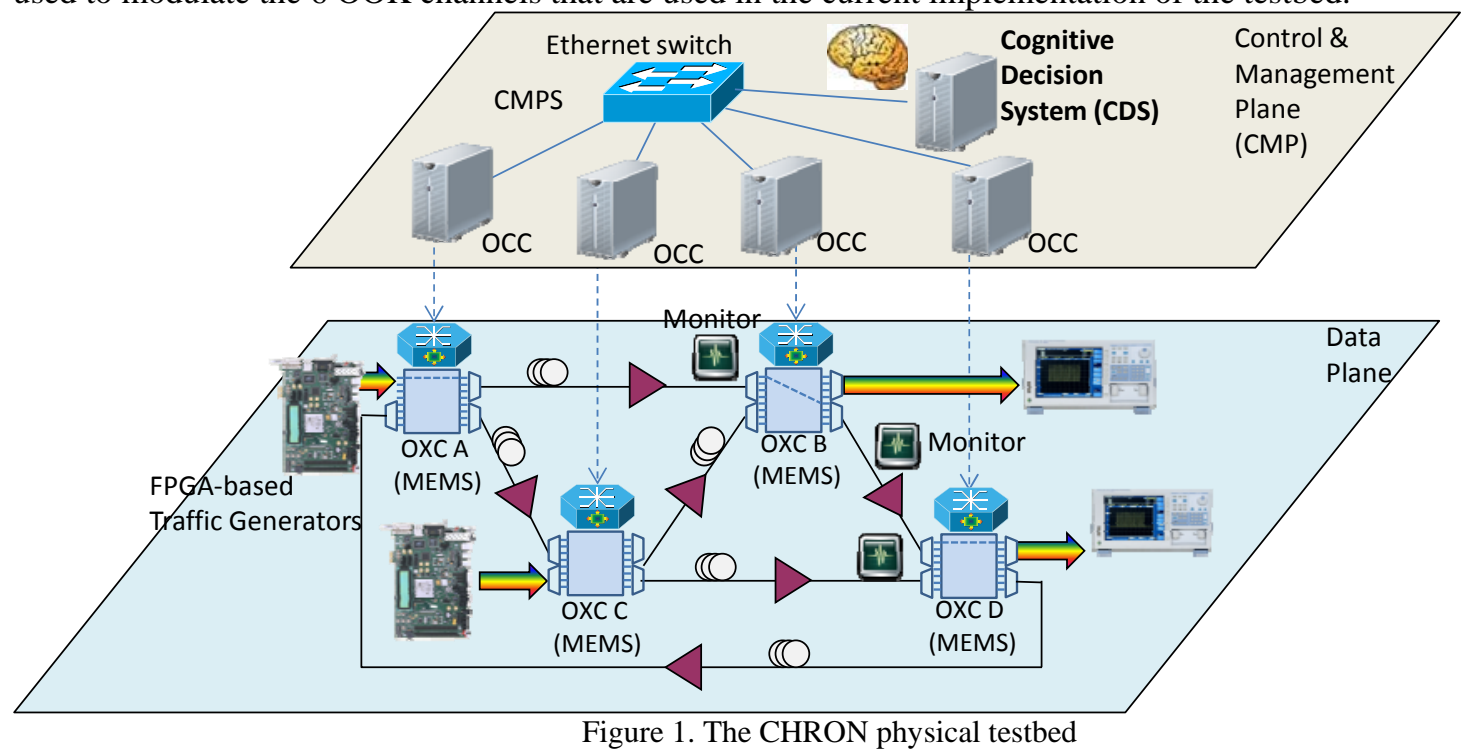

To demonstrate the operation of the CHRON testbed one of the testing scenarios is as follows:

1. A lightpath is established between nodes A and D going through node B (i.e., the shortest path).

2. The fiber link between A and B is cut. Several power and BER monitors trigger low power/BER alarms, which are retrieved by the CDS thanks to the CMP.

3. The CDS evaluates the alarms and identifies that an AB link failure event has taken place. It then chooses a suitable restoration action. As the failure is a fibre cut (and not "simply” a Quality of Transmission -QoTdegradation), changing the modulation format is not a suitable solution. Then, the CDS initiates a link 
restoration procedure. First of all, its internal QoT estimator module assesses the QoT of path ACBD and it estimates that the QoT will be fine. Then it instructs the CMP to setup the restoration lightpath through path ACBD.

4. However, the QoT estimator was wrong, and the new connection has high BER. Therefore, a network monitor associated to the lightpath receiver triggers a low quality (high BER) alarm, which is notified to the CDS by means of the CMP.

5. Then, the CDS learns from this failure and the QoT estimator module updates his knowledge base with this information. Moreover, the CDS takes a corrective action, determining that path restoration should be employed and path ACD should be used instead (which is also verified by the QoT estimator module). The CMP is then used to reconfigure the MEMS switches in order to release the previous lightpath and establish it through the new route. The real QoT of this new path is very high (low BER) so no alarms are triggered, but monitor data confirms to the CDS that its QoT assessment was correct.

In the following steps, we verify that the CDS has learnt from the past and thus improved its performance.

6. The established lightpath in the testbed is released, and steps 1 and 2 are repeated.

7. As the CDS has learnt that path ACBD does not produce enough QoT, it directly triggers the path restoration procedure through route ACD in order to restore the lightpath between A and D. In this way, the restoration time is reduced when compared with the previous situation.

The operation of this scenario is depicted together with timing results in Figure 2. As it is shown in this figure, the use of the CDS reduces the time of the link restoration by over $48 \%$.

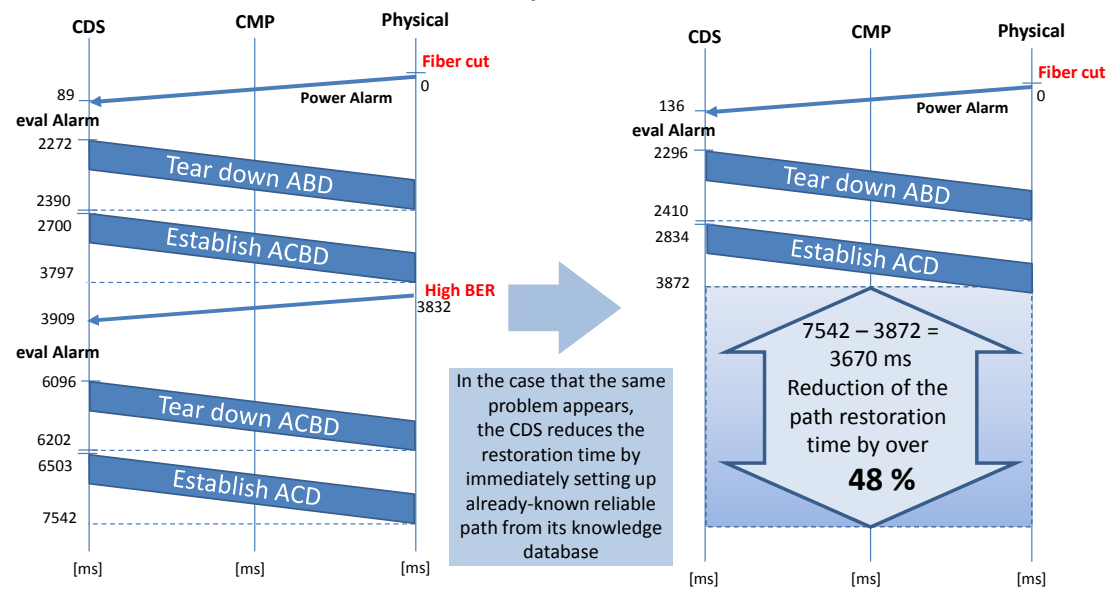

Figure 2. Reduction of the path restoration using the CHRON Cognitive Decision System (CDS)

\section{Conclusions}

The developed physical testbed demonstrates the realization of an advanced cognitive engine that is targeting future heterogeneous reconfigurable optical networks. The implemented physical testbed does not only demonstrate the operation of the cognitive techniques that has been developed in a real network but it also demonstrates the integration of all the required modules such as control and management plane, data plane, etc. to make this scheme applicable to current and future optical networks. In particular, we have demonstrated a simple case of lightpath restoration in a 4 nodes testbed, where by exploiting cognitive techniques, a reduction of up to $48 \%$ restoration time can be achieved. Nevertheless, the performance evaluation of the cognitive engine in realistic networks using a large number of nodes has been shown in [4].

\section{Acknowledgments}

The research leading to these results is partially supported by the CHRON project (Cognitive Heterogeneous Reconfigurable Optical Network) with funding from the European Community's Seventh Framework Programme [FP7/2007-2013] under grant agreement $n^{\circ}$ 258644. We thank Xilinx University Program (XUP) for the kind donation of Nexys2 Boards and the EDA tools.

\section{References}

[1] R.W.Thomas et al,Cognitive networks: Adaptation and learning to achieve end-to-end performance objectives, Comm. Mag(51-57),Dec’06

[2] Q.H. Mahmoud (ed.), Cognitive Networks: Towards Self-Aware Networks (John Wiley \& Sons, Ltd., 2007).

[3] I. Tomkos et al., "Next generation flexible and cognitive heterogeneous optical networks," in The Future Internet, 2012 , pp. 225-236.

[4] T. Jiménez et al., “A Cognitive Quality of Transmission Estimator for Core Optical Networks,”, JLT, Issue 31, pp. 942-951, 2013

[5] I. de Miguel et al. "Cognitive Dynamic Optical Networks [Invited]," JOCN, Vol. 5, Issue 10, pp. A107-A118 Oct. 1, 2013.

[6] D. Siracusa, et al., "A Control Plane Framework for Future Cognitive Heterogeneous Optical Networks”, ICTON 2012

[7] OMM 8x8-2 MEMS Optical Cross Connect Switch (OXC), OMM Inc., Datasheet

[8] Transceiver Signal Integrity Development Kit, Stratix IV GT Edition, Altera Inc., Datasheet 